\title{
Desigualdades de género en la distribución de los recursos económicos en las parejas
}

\author{
Capitolina Díaz Martínez
}

Universidad de Valencia. Departamento de Sociología y Antropología Social

capitolina.diaz@uv.es

\section{Sandra Dema Moreno}

Universidad de Oviedo. Departamento de Sociología

demasandra@uniovi.es

\section{Lucila Finkel}

Universidad Complutense de Madrid, Departamento de Sociología IV

(Metodología de la Investigación Social y Teoría de la Comunicación)

lfinkelm@ucm.es

\section{Resumen}

En este artículo, se analizan las desigualdades de género que se producen en los hogares españoles, particularmente en los formados por parejas de doble ingreso. A partir del estudio de los microdatos de la Encuesta de Condiciones de Vida (ECV), se establece una tipología de hogares en función de las características diferenciales de las parejas que los componen, atendiendo a la ocupación, la actividad, la edad, los ingresos y los niveles de estudio. El análisis se completa con una descripción detallada de las características de las parejas de doble ingreso, haciendo un énfasis especial en las diferencias y en las desigualdades internas entre sus miembros en relación con algunas de las variables consideradas. Se estima, asimismo, la probabilidad de que las mujeres presenten mayores ingresos que los hombres, a través de un análisis de regresión logística. Por último, el artículo ofrece algunas consideraciones sobre las desigualdades económicas presentes en hogares unipersonales y monoparentales.

Palabras clave: ingresos; relaciones de género; hogares; parejas de doble ingreso; análisis de conglomerados; análisis de regresión logística.

\section{Abstract. Gender inequalities in the distribution of financial resources within couples}

This article focuses on gender inequalities that occur in Spanish households, particularly those formed by dual-income couples. From the analysis of the Spanish version of the European Union Survey on Income and Living Conditions (EU-SILC) microdata, we establish a typology of households based on the different characteristics of the couples, 
taking into account occupation, activity status, age, income, and educational level. The analysis is followed by a detailed description of the characteristics of the dual-income couples with an emphasis on the internal differences and inequalities between partners with respect to some of the variables considered. The likelihood that women earn higher incomes than men is estimated through a logistic regression analysis. Finally, the paper puts forward some considerations about economic inequalities present in one-person and single-parent households.

Keywords: income; gender relations; households; dual-income couples; cluster analysis; logistic regression analysis

\section{Sumario}

1. Introducción 6. Desigualdades económicas entre

2. Metodología hombres y mujeres en otros tipos de hogares

3. Caracterización de las parejas

4. El aumento de las parejas de doble ingreso

7. Conclusiones

Referencias bibliográficas

5. Desigualdades económicas en las parejas de doble ingreso.

La dependencia intrapareja

\section{Introducción ${ }^{1}$}

Cualquier investigación sobre la familia en España exige tener en cuenta los principales cambios sociales y políticos que ha experimentado nuestra sociedad en las últimas tres décadas y, particularmente, las transformaciones que conciernen a esta institución social (Del Campo, 1991; Jurado y Naldini, 1996; Alberdi, 1999; Meil, 1999; González y Requena, 2005). Una evolución que está afectando a la estructura de las familias, a sus funciones sociales y a sus propias dinámicas internas.

Para entender tales cambios, las transformaciones en las relaciones de género son uno de los elementos centrales. Las mujeres han accedido progresivamente a la educación, han alcanzado e incluso superado el nivel educativo de

1. Este artículo ha sido realizado en el seno del proyecto de investigación Los presupuestos familiares desde la perspectiva de género: Análisis no sexista de la Encuesta de Presupuestos Familiares y de la Encuesta de Condiciones de Vida (CSO2008-05182), financiado por el Plan Nacional de I+D+i (2008-2011), del Ministerio de Educación y Ciencia. Asimismo, ha recibido una ayuda de cofinanciación por parte de la Consejería de Educación y Ciencia del Principado de Asturias (FC09COF0922). Una versión previa del mismo ha sido presentada en el seminario Wealth and poverty in personal relationships, en el Instituto Internacional de Sociología Jurídica, en mayo de 2012. Agradecemos a nuestras compañeras de proyecto Marta Ibáñez Pascual y Benita Compostela Muñiz su valiosa labor investigadora a lo largo de estos años, así como a Inés Villamil Rico su participación en el análisis de los microdatos de la Encuesta de Condiciones de Vida. 
los varones en las generaciones más jóvenes y se han incorporado masivamente al trabajo remunerado, aunque su tasa de actividad es inferior a la de los varones españoles (un 53,4\% la femenina frente a un 65,6\% la masculina en 2013). Asimismo, las mujeres españolas tienen una tasa de ocupación del 39,1\%, ligeramente inferior a la de las europeas (del $45,6 \%$ en los 28 países de la UE).

Estas transformaciones en la esfera educativa y laboral han venido acompañadas de importantes cambios en el ámbito doméstico, como el retraso en la edad de contraer matrimonio y en la de tener descendencia ${ }^{2}$. Los valores familiares también están en un momento de evolución y se observa una cierta democratización de los mismos, especialmente en las generaciones más jóvenes. El estudio Jóvenes e igualdad de género, llevado a cabo en 2008 por el Instituto de la Juventud (INJUVE), muestra que el 84,6\% de los jóvenes y las jóvenes considera que el modelo ideal de pareja es aquel en el que ambos miembros tienen un empleo y comparten el trabajo doméstico y de cuidado de personas dependientes en igualdad de condiciones, mientras que el 9,6\% preferiría que la mujer trabajara menos horas fuera de casa y asumiera mayoritariamente el trabajo doméstico y de cuidado en el interior del hogar. Sólo el 4,3\% de las jóvenes y los jóvenes prefieren el modelo tradicional de varón proveedor y mujer ama de casa (INJUVE, 2008).

Estas transformaciones de la práctica social y del mundo de los valores se reflejan en la diversidad de tipos de hogares: la familia nuclear tradicional de varón proveedor y mujer ama de casa convive con otros modelos más frecuentes hasta ahora en el resto de países de nuestro entorno, como las parejas de doble ingreso, las familias monoparentales, las familias multiétnicas, las parejas de hecho y las uniones homosexuales, entre otras. Las parejas de doble ingreso constituyen una forma de convivencia cada vez más frecuente en nuestra sociedad, de hecho, han aumentado claramente en las dos últimas décadas.

Las desigualdades de género en las familias tradicionales solían residir en la radical división sexual del trabajo tanto en el interior del hogar como en el empleo. El hecho de que, hoy en día, las mujeres contribuyan cada vez más a los ingresos familiares y tengan una vida profesional propia, en principio, podría ser considerado un buen punto de partida para el establecimiento de unas relaciones más igualitarias en el interior del hogar. Nuestro interés se centra en conocer en qué medida las desigualdades de género perviven en las parejas de doble ingreso y si la diferente aportación económica de cada miembro de la pareja tiene un efecto perceptible en el aumento o disminución de la igualdad en el interior de los hogares.

Las desigualdades económicas que se generan en el hogar son el resultado de una amplia combinación de factores tales como las discriminaciones

2. Los datos demográficos muestran también una considerable reducción en la tasa de natalidad, no sólo en España, sino también en la mayoría de los países del sur de Europa. Esto puede ser interpretado como una estrategia, implementada no de forma intencional y colectiva, sino más bien de forma individual por las mujeres y los varones de estos países, con el fin de impedir un empeoramiento de su nivel de vida, reduciendo en lo posible sus cargas familiares (Alberdi, 2006: 31). 
laborales que sufren las mujeres (Villar, 2010; Torns y Recio, 2013), el reflejo de la brecha salarial ${ }^{3}$ en el interior de las familias (Dema, 2011), el tipo de políticas públicas, en particular de las relativas a la conciliación de la vida laboral y personal (Daly y Lewis, 2000; Torns, 2005) y a la oferta de atención a las personas dependientes (Carrasco y Rodríguez, 2000; Carrasco, 2003; Tobío, 2005; Larrañaga et al., 2008; Torns y Recio, 2013), junto con la pervivencia de una cultura que asocia los varones a lo monetario y las mujeres a los cuidados.

En este artículo, vamos a analizar las diferencias económicas entre mujeres y varones en los hogares españoles. Seguiremos una línea teórica de la sociología de la familia que ha resultado muy fecunda en las últimas décadas. Efectivamente, desde los años sesenta, han aparecido numerosos estudios que sugieren que el dinero puede ser un buen indicador para comprender las relaciones de género en las familias contemporáneas (Blood y Wolfe, 1960; Pahl, 1989; Vogler y Pahl, 1994; Zelizer, 1997; Tichenor, 1999). En nuestro país, estos estudios son mucho más recientes y se han centrado en entender cuál es el significado que varones y mujeres otorgan al dinero que ganan (Dema y Díaz, 2010), cómo son los modelos de gestión y administración del dinero de las parejas españolas (Dema, 2006; Stocks et al., 2007) y cómo toman las decisiones de carácter económico las parejas de doble ingreso (Dema, 2009).

\section{Metodología}

La base empírica de este artículo proviene de la Encuesta de Condiciones de Vida (ECV), que permite identificar la manera en la que se distribuyen los recursos económicos entre mujeres y hombres en el interior de los hogares españoles ${ }^{4}$. Se parte de los datos recogidos por el Instituto Nacional de Estadística

3. La brecha salarial, esto es, la diferencia retributiva entre varones y mujeres, ha sido objeto de numerosos estudios en el ámbito internacional desde la década de los setenta (Oaxaca, 1973; Blinder, 1973; Neumark, 1988). En España, los primeros estudios son más recientes, pero, en los últimos años, ha habido numerosas investigaciones sobre esta cuestión (De Cabo y Garzón, 2007; Sallé y Molpeceres, 2010; Villar, 2010). En la mayor parte de dichos estudios, además de ofrecer una visión de la magnitud y la evolución del fenómeno, se ha hecho hincapié en las formas de medir la brecha salarial y en las principales causas que podrían explicarla.

4. La Encuesta de Condiciones de Vida (ECV), del Instituto Nacional de Estadística (INE), se realiza en España desde el año 2004 y se lleva a cabo de forma armonizada en todos los países de la Unión Europea, donde se conoce como EU-SILC (Community Statistics on Income and Living Conditions). Aunque, fundamentalmente, proporciona datos sobre pobreza y exclusión, también permite obtener información de gran interés sobre ingresos en los hogares, empleo y actividad, vivienda, nivel de formación y salud, entre otras cuestiones. Se trata de una encuesta de tipo "panel rotante», en la que se investiga a las mismas personas durante cuatro años, aunque van rotando anualmente. Para mayor detalle sobre su metodología, puede consultarse la documentación del INE:

<http://www.ine.es/dynt3/metadatos/es/RespuestaDatos.htm?oe=30453.> 
en $2008^{5}$ a una muestra de 13.014 hogares, aunque ha sido necesario realizar una serie de transformaciones previas que han permitido obtener una base de datos adecuada a los propósitos de este artículo ${ }^{6}$.

De estos hogares, hemos estudiado especialmente aquellos formados por parejas heterosexuales, con o sin menores dependientes ${ }^{7}$, y que no conviven con otras personas adultas o económicamente activas. Aunque coincidimos con algunas investigaciones que sugieren el interés de analizar parejas homosexuales y compararlas con las heterosexuales (Clarke et al., 2005), el escaso porcentaje de las primeras en la muestra $(0,2 \%$ de la ECV) ha impedido dicha comparación.

A partir de las variables individuales y del hogar recogidas a través de la ECV, hemos creado nuevas variables que contrastan y combinan la información proporcionada por ambos miembros de la pareja y permiten sintetizar características sociodemográficas individuales en nuevas variables agregadas que nos sirven para caracterizar el tipo de pareja de que se trate.

En primer lugar, y dado que las condiciones de vida y los ingresos de las personas vienen fuertemente determinados por el momento del ciclo vital en el que se encuentran, hemos categorizado los hogares en tres generaciones: parejas jóvenes, adultas y mayores. La categoría parejas jóvenes incluye aquellas en los que ambos miembros de la pareja tienen entre 16 y 34 años. Este grupo constituye el $15 \%$ del total. Hemos establecido los 16 años como extremo inferior del intervalo, dado que es cuando empieza la edad laboral en España, y hemos limitado el intervalo a 34 años, considerando la edad de emancipación y primer matrimonio, así como la edad media en la que se tiene el primer hijo, que está en torno a los 30 años, tanto para mujeres como para hombres.

5. Las relaciones de género en el interior de los hogares no varían demasiado en términos anuales, dado que responden a tendencias que se consolidan a lo largo del tiempo, de ahí que no sea previsible que, entre el año estudiado y el actual, se hayan experimentado grandes cambios, si acaso, podríamos encontrar una mayor desigualdad de género consecuencia de la crisis y de las políticas de recortes sobre la situación de las mujeres. Por tanto, cabe advertir que, por tratarse de una foto fija de un año, se ha realizado un análisis de carácter transversal, que no pretende medir evoluciones históricas ni tendencias, por lo que no cabría inferir, por ejemplo, que el incremento observado de parejas igualitarias más jóvenes se mantenga cuando éstas alcancen mayor edad.

6. El Instituto Nacional de Estadística ofrece los microdatos de la ECV en cuatro archivos: datos básicos del hogar (fichero D), datos básicos de la persona (fichero R), datos detallados del hogar (fichero H) y datos detallados de los adultos (fichero P). Con el fin de obtener un fichero de datos detallados de parejas que conviven en el mismo hogar, se han fusionado los ficheros $\mathrm{H}$ y P, extrayendo únicamente los hogares formados por parejas y desechando los unipersonales y los monoparentales. El fichero resultante contiene variables que hacen referencia al hogar, pero también variables individuales de los miembros de la pareja que han sido codificadas con .1 para los hombres y .2 para las mujeres. El total de la muestra es de 6.154 hogares con parejas con y sin hijos, aunque se ha ponderado la muestra por el factor de ponderación transversal del hogar del INE, lo que arroja un total de 8.007.756 casos. Por tanto, tomamos como unidad de análisis el hogar, aunque se consideran también variables relativas a individuos.

7. Entendiendo como dependientes a aquellas personas menores de 25 años que son laboralmente inactivas. 
Hemos tenido en cuenta, además, que, en este rango de edad, hay un lapso de tiempo suficiente para que las parejas puedan establecer pautas económicas que, potencialmente, arrojen información acerca de sus relaciones de género.

La categoría parejas adultas incluye aquellas en las que la persona de mayor edad (sea el hombre o la mujer) tiene entre 35 y 64 años. Este grupo concentra el $64 \%$ de la muestra.

Por último, la categoría parejas mayores representa el $21 \%$ restante e incluye a aquellas en las que el miembro mayor tiene 65 o más años. En este rango de edad, la mayoría de las personas ha abandonado el mercado laboral y sus ingresos provienen de las pensiones.

En segundo lugar, se han combinado variables sociodemográficas individuales, como ocupación, actividad o ingresos, para construir variables que permitan categorizar a los hogares en función de si son de doble ingreso o no, o si ambos miembros de la pareja o sólo uno de ellos está ocupado o en situación de actividad.

En tercer lugar, se han creado nuevas variables que sintetizan las diferencias entre el hombre y la mujer en lo que respecta al nivel educativo (determinando quién tiene un nivel educativo más elevado o si es igual), a los ingresos ${ }^{8}$ (si uno de los integrantes de la pareja aporta más o si ambos aportan una cantidad similar) y a los porcentajes de diferencia en los ingresos de ambos miembros de la pareja (teniendo en cuenta cuál sería dicho porcentaje).

El análisis estadístico realizado parte de los análisis descriptivos de estas variables, pero pone un énfasis especial en los resultados obtenidos a partir de dos técnicas de análisis multivariantes (análisis de conglomerados y análisis de regresión logística), que permiten tratar las influencias y las interacciones simultáneas de las variables descritas de los hogares españoles que forman parte de la muestra.

Asimismo, hemos comparado las desigualdades económicas entre diversos tipos de hogares, ya que las parejas constituyen un tipo de convivencia que permite observar con claridad las diferencias entre varones y mujeres en el interior de las familias y compararlas con los ingresos de varones y mujeres en otros modelos familiares -como los hogares unipersonales y los hogares monoparentales encabezados por mujeres (dado que apenas existen hogares monoparentales encabezados por varones, no se les ha podido incluir en la comparación).

8. En el caso de los ingresos del hogar, que corresponden al año anterior a la realización de la encuesta, se ha considerado la variable renta bruta total del hogar (HY010). Para calcular los ingresos brutos anuales de la persona, se ha construido una nueva variable a partir de la suma de la renta bruta monetaria o cuasi monetaria, la renta bruta no monetaria, los beneficios o las pérdidas monetarias brutas de trabajadores por cuenta propia, las rentas brutas percibidas de planes de pensiones privados, las prestaciones brutas por desempleo, supervivencia, jubilación, enfermedad o incapacidad, así como la ayuda bruta para estudios. A partir de los ingresos brutos del hombre y de la mujer, se han calculado las diferencias de ingresos entre la pareja, así como el porcentaje que cada uno de sus miembros aporta a la unidad familiar. 


\section{Caracterización de las parejas}

Una primera aproximación a los datos descriptivos de la ECV en lo relativo a las variables de pareja consideradas hacía intuir la importancia de establecer una tipología de las clases de hogares existentes en función de estas variables, por lo que se llevó a cabo un análisis de conglomerados ${ }^{9}$ que ha permitido identificar tres grandes grupos distribuidos de forma relativamente homogénea, como muestra la figura 1 .

El análisis de las asociaciones bivariadas de los conglomerados resultantes con las distintas categorías de las variables consideradas nos permite observar las características más relevantes de las parejas que conforman la muestra. La tabla 1 incluye los porcentajes obtenidos de los cruces bivariados con sus coeficientes de asociación lambda correspondientes (se resaltan en negrita los porcentajes más significativos).

Se observa que todas las variables consideradas presentan niveles estadísticos de asociación lambda relevantes, excepto el riesgo de pobreza, que, efectivamente, arroja un lambda igual a cero. En el resto de los casos, se observan porcentajes diferenciados en un conglomerado u otro, que, considerados de forma conjunta, nos permiten resumir las características principales del tipo de pareja que puede identificarse en cada conglomerado:

\section{Conglomerado 1 (46\%): Pareja adulta tradicional ${ }^{10}$}

- Ambos ocupados o él ocupado y ella no.

- Ambos activos.

Figura 1. Distribución de la muestra en tres conglomerados ( $n=6.154$ hogares)

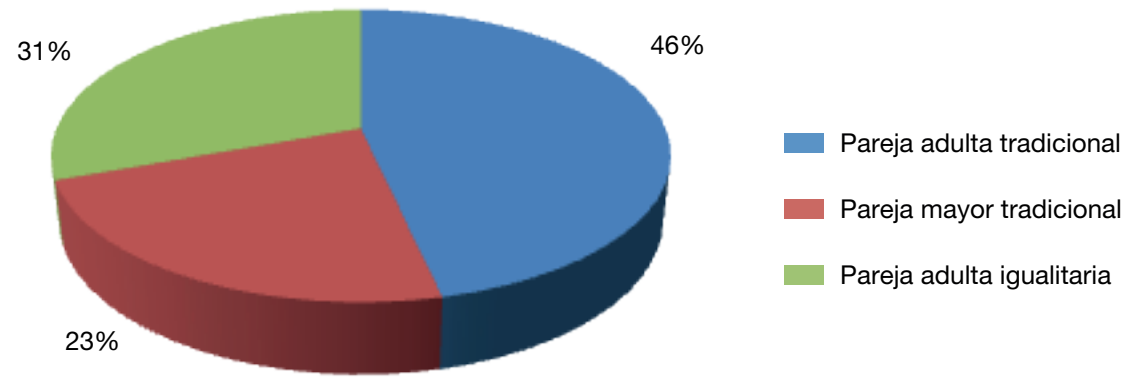

Fuente: elaboración propia a partir de los microdatos de la ECV 2008.

9. Análisis de conglomerados de $\mathrm{K}$ medias a partir de variables originales estandarizadas para minimizar el efecto de los distintos niveles de medición. Centroides originales y finales diferenciados en los distintos conglomerados, que tienen los siguientes tamaños (1: 2624, 2: 1670 y $3: 1624)$.

10. Por «pareja tradicional» entendemos el clásico modelo de varón proveedor y mujer ama de casa o bien a parejas de doble ingreso en las que el salario masculino es sustancialmente más elevado que el femenino. 
Tabla 1. Caracterización de los distintos conglomerados

\begin{tabular}{|c|c|c|c|}
\hline & \multicolumn{3}{|c|}{ Conglomerados } \\
\hline & 1 & 2 & 3 \\
\hline \multicolumn{4}{|l|}{ Ocupación $(\lambda=0,44)$} \\
\hline Él ocupado y ella no & $47,6 \%$ & $0,0 \%$ & $2,1 \%$ \\
\hline Ambos ocupados & $50,3 \%$ & $0,0 \%$ & $86,1 \%$ \\
\hline Ella ocupada y él no & $0,7 \%$ & $2,6 \%$ & $9,8 \%$ \\
\hline Ninguno ocupado & $1,3 \%$ & $97,4 \%$ & $2,0 \%$ \\
\hline Total & $100,0 \%$ & $100,0 \%$ & $100,0 \%$ \\
\hline \multicolumn{4}{|l|}{ Actividad $(\lambda=0,48)$} \\
\hline Él activo y ella no & $36,4 \%$ & $1,2 \%$ & $1,5 \%$ \\
\hline Ambos activos & $63,3 \%$ & $1,3 \%$ & $93,0 \%$ \\
\hline Ella activa y él no & $0,3 \%$ & $7,4 \%$ & $4,9 \%$ \\
\hline Ninguno activo & $0,0 \%$ & $90,1 \%$ & $0,7 \%$ \\
\hline Total & $100,0 \%$ & $100,0 \%$ & $100,0 \%$ \\
\hline \multicolumn{4}{|l|}{ Miembro que ingresa $(\lambda=0,12)$} \\
\hline Ingresa solo él & $37,0 \%$ & $58,5 \%$ & $0,1 \%$ \\
\hline Ingresan ambos & $62,7 \%$ & $41,5 \%$ & $94,3 \%$ \\
\hline Ingresa solo ella & $0,3 \%$ & $0,0 \%$ & $5,6 \%$ \\
\hline Total & $100,0 \%$ & $100,0 \%$ & $100,0 \%$ \\
\hline \multicolumn{4}{|l|}{ Miembro que aporta más $(\lambda=0,5)$} \\
\hline Él aporta más & $98,7 \%$ & $82,7 \%$ & $8,5 \%$ \\
\hline Aportan igual & $1,3 \%$ & $16,0 \%$ & $62,3 \%$ \\
\hline Ella aporta más & $0,0 \%$ & $1,3 \%$ & $29,1 \%$ \\
\hline Total & $100,0 \%$ & $100,0 \%$ & $100,0 \%$ \\
\hline \multicolumn{4}{|l|}{ Nivel educativo de la pareja $(\lambda=0,15)$} \\
\hline Nivel educativo igual & $50,2 \%$ & $65,6 \%$ & $57,3 \%$ \\
\hline Él mayor nivel educativo & $25,9 \%$ & $23,2 \%$ & $13,5 \%$ \\
\hline Ella mayor nivel educativo & $24,0 \%$ & $11,2 \%$ & $29,2 \%$ \\
\hline Total & $100,0 \%$ & $100,0 \%$ & $100,0 \%$ \\
\hline \multicolumn{4}{|l|}{ Diferencia de aportación $(\lambda=0,39)$} \\
\hline Ella aporta más del $75 \%$ más que él & & $1,0 \%$ & $0,2 \%$ \\
\hline Ella aporta entre el 46 y el $74 \%$ más que él & & $5,1 \%$ & $0,6 \%$ \\
\hline Ella aporta entre el 16 y el $45 \%$ más que él & & $20,7 \%$ & $2,9 \%$ \\
\hline Aportan igual (diferencia hasta el 15\%) & $2,1 \%$ & $73,2 \%$ & $40,6 \%$ \\
\hline Él aporta entre el 16 y el $45 \%$ más que ella & $56,9 \%$ & & $30,7 \%$ \\
\hline Él aporta entre el 46 y el $74 \%$ más que ella & $30,7 \%$ & & $20,7 \%$ \\
\hline Él aporta más del $75 \%$ más que ella & $10,3 \%$ & & $4,3 \%$ \\
\hline Total & $100,0 \%$ & $100,0 \%$ & $100,0 \%$ \\
\hline \multicolumn{4}{|l|}{ Tramos de edad $(\lambda=0,3)$} \\
\hline Pareja joven (16-34) & $19,0 \%$ & $0,2 \%$ & $20,6 \%$ \\
\hline Pareja adulta (35-44) & $44,0 \%$ & $1,5 \%$ & $40,9 \%$ \\
\hline Pareja adulta (45-54) & $26,1 \%$ & $1,5 \%$ & $26,2 \%$ \\
\hline Pareja adulta (55-64) & $9,7 \%$ & $10,4 \%$ & $9,4 \%$ \\
\hline Pareja mayor (65 o más) & $1,1 \%$ & $86,4 \%$ & $2,9 \%$ \\
\hline Total & $100,0 \%$ & $100,0 \%$ & $100,0 \%$ \\
\hline \multicolumn{4}{|l|}{ Menores en el hogar $(\lambda=0,46)$} \\
\hline Pareja sin menores & $24,9 \%$ & $95,4 \%$ & $37,8 \%$ \\
\hline Pareja con menores & $75,1 \%$ & $4,6 \%$ & $62,2 \%$ \\
\hline Total & $100,0 \%$ & $100,0 \%$ & $100,0 \%$ \\
\hline \multicolumn{4}{|l|}{ Hogar en riesgo de pobreza $(\lambda=0,0)$} \\
\hline No & $83,5 \%$ & $68,7 \%$ & $92,8 \%$ \\
\hline Sí & $16,5 \%$ & $31,3 \%$ & $7,2 \%$ \\
\hline Total & $100,0 \%$ & $100,0 \%$ & $100,0 \%$ \\
\hline
\end{tabular}

$p<0,000$ en todos los casos. 
- Ingresan ambos.

- Él aporta más que ella (en todos los tramos considerados).

- Mismo nivel educativo.

- Pareja entre 35 y 44 años.

- Hay menores en el hogar.

Conglomerado 2 (23\%): Pareja mayor tradicional

- Ninguno ocupado.

- Ninguno activo.

- Prevalecen ligeramente los hogares donde ingresa sólo él.

- El aporta más que ella, pero las diferencias son en gran parte inferiores al 15\%.

- Mismo nivel educativo.

- Pareja de 65 o más años.

- No hay menores en el hogar.

Conglomerado 3 (31\%): Pareja adulta igualitaria ${ }^{11}$

- Ambos ocupados.

- Ambos activos.

- Ingresan ambos.

- Aportan por igual o él aporta entre un 16\% y un $45 \%$ más.

- Mismo nivel educativo o ella mayor nivel educativo.

- Pareja entre 35 y 44 años.

- Hay menores en el hogar.

\section{El aumento de las parejas de doble ingreso}

El número de parejas españolas de doble ingreso se ha incrementado sustancialmente en los últimos años. En 1992, se estimaba que en sólo un tercio de los hogares formados por parejas integradas por personas entre 20 y 59 años entraban dos salarios, mientras que, en el 2002, ya eran el $45 \%$ de dichos hogares los que recibían dos ingresos (Franco y Winqvist, 2002). Según los datos de la Encuesta de Condiciones de Vida (ECV) de 2008, el 68\% de las parejas son de doble ingreso, mientras que, en el 30\%, se mantiene el modelo tradicional de varón proveedor y mujer ama de casa (que se identifica fundamentalmente con el conglomerado 2) y, en el $2 \%$ restante, sólo la mujer percibe ingresos.

Si tenemos en cuenta la edad de las parejas, el 85\% de las jóvenes (de 16 a 34 años) son de doble ingreso, mientras que menos del $45 \%$ de las mayores de 65 años lo son. En las parejas mayores de 65 años, el modelo mayoritario es el tradicional, en el que sólo tiene ingresos el varón. Un modelo que practica el $55 \%$ de estas parejas mayores, frente al 12\% de las parejas jóvenes (figura 2).

11. Por "pareja igualitaria» nos referimos a aquellas en las que ambos miembros del hogar aportan una cantidad de ingresos semejante o bien la diferencia a favor del varón es menor que en el modelo anterior. 
Figura 2. Porcentaje de hogares según el miembro de la pareja que recibe ingresos, por edad de la pareja $(n=6.154$ hogares)

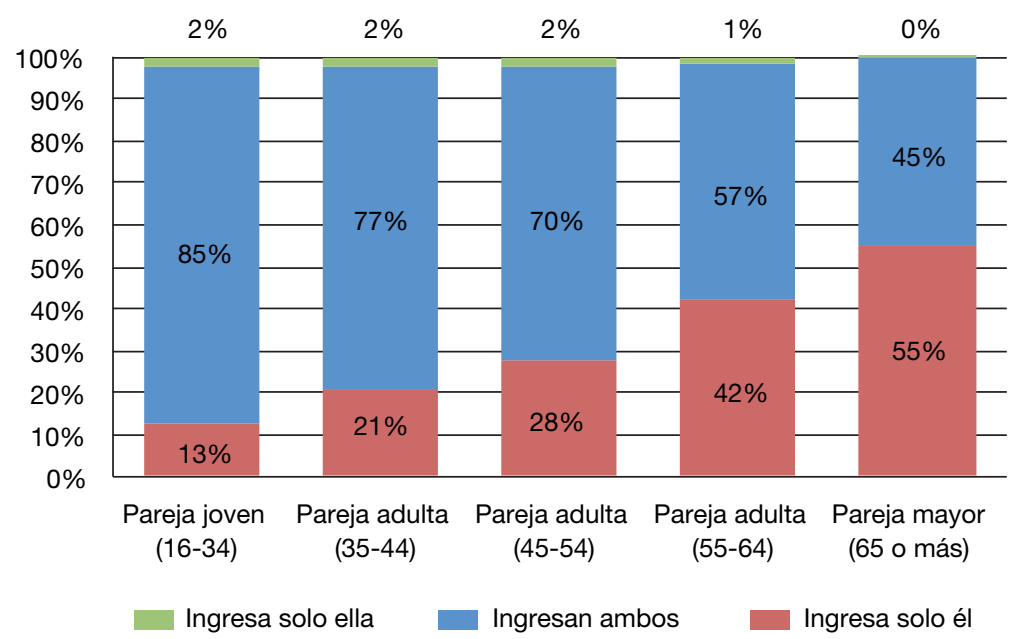

Fuente: elaboración propia a partir de los microdatos de la ECV 2008.

Figura 3. Nivel educativo según edad de la pareja ( $n=6.154$ hogares)

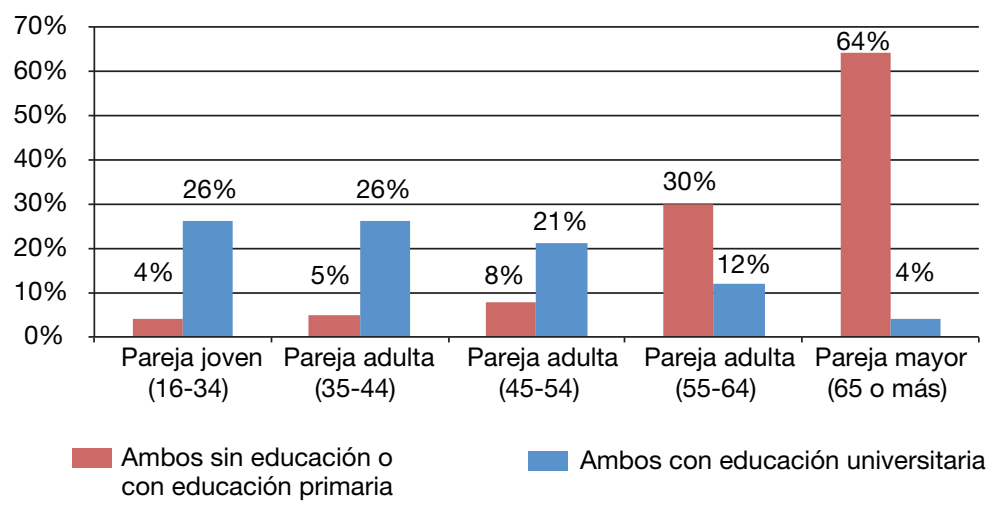

Fuente: elaboración propia a partir de los microdatos de la ECV 2008.

El aumento de las parejas de doble ingreso está relacionado, sobre todo, con la mejora del nivel educativo de las mujeres en las últimas décadas y su entrada en el mercado laboral. El acceso al sistema educativo, tanto de varones como de mujeres, se ha incrementado notablemente en las últimas décadas (figura 3). 
Figura 4. Miembro de la pareja con mayor nivel educativo, según la edad de la pareja ( $n=6.154$ hogares)

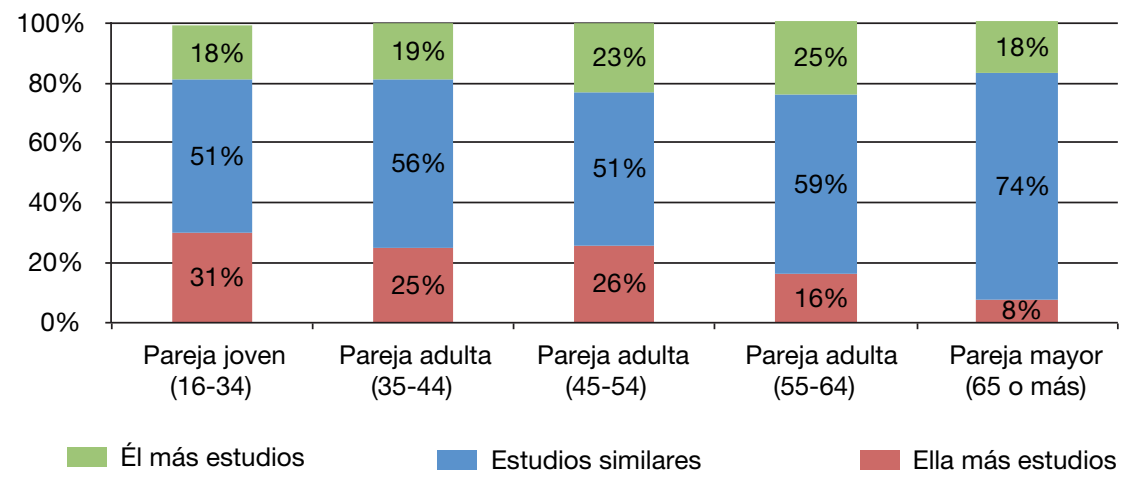

Fuente: elaboración propia a partir de los microdatos de la ECV 2008.

En el 64\% de las parejas mayores (65 años o más), ninguno de los dos miembros tiene estudios o únicamente han superado los primarios, mientras que sólo el 4\% de las parejas más jóvenes se ha quedado en el nivel de educación primaria. Por el contrario, en torno al 26\% de las parejas más jóvenes (de 16 a 34 años), ambos miembros tienen estudios universitarios, mientras que en las mayores sólo se da esta situación en el 4\% de los casos. Esto es, el nivel educativo desciende a medida que aumenta la edad de las parejas.

Es destacable la fuerte homogamia encontrada, puesto que el 58,6\% de las parejas tiene el mismo nivel de estudios (figura 4), si bien en las generaciones más jóvenes hay un mayor porcentaje de mujeres con un nivel de estudios superior al de sus parejas, al contrario de lo que ocurre en las generaciones de mayor edad.

El aumento de las parejas de doble ingreso a lo largo de las últimas décadas está claramente vinculado al acceso de las mujeres al mercado laboral. La figura 5 pone de manifiesto cómo, en las parejas más jóvenes, casi el $80 \%$ de las mujeres tiene un empleo, una tendencia que se reduce progresivamente con la edad. Por el contrario, el porcentaje de mujeres que nunca ha tenido un trabajo remunerado aumenta sobre todo a partir de los 55 años. Entre las parejas menores de 55 años, el porcentaje de mujeres que nunca han trabajado de forma remunerada (tanto en las adultas como en las jóvenes) es relativamente bajo, inferior al 6\%, mientras que entre las mayores de 65 años alcanza el 35\%.

Sin embargo, varones y mujeres no han accedido al mercado laboral en las mismas circunstancias, tanto por la propia socialización diferenciada por género, como por los persistentes estereotipos de género (histéresis 
Figura 5. Porcentaje de hombres y de mujeres en distintas situaciones laborales, según la edad de la pareja ( $n=6.154$ hogares)

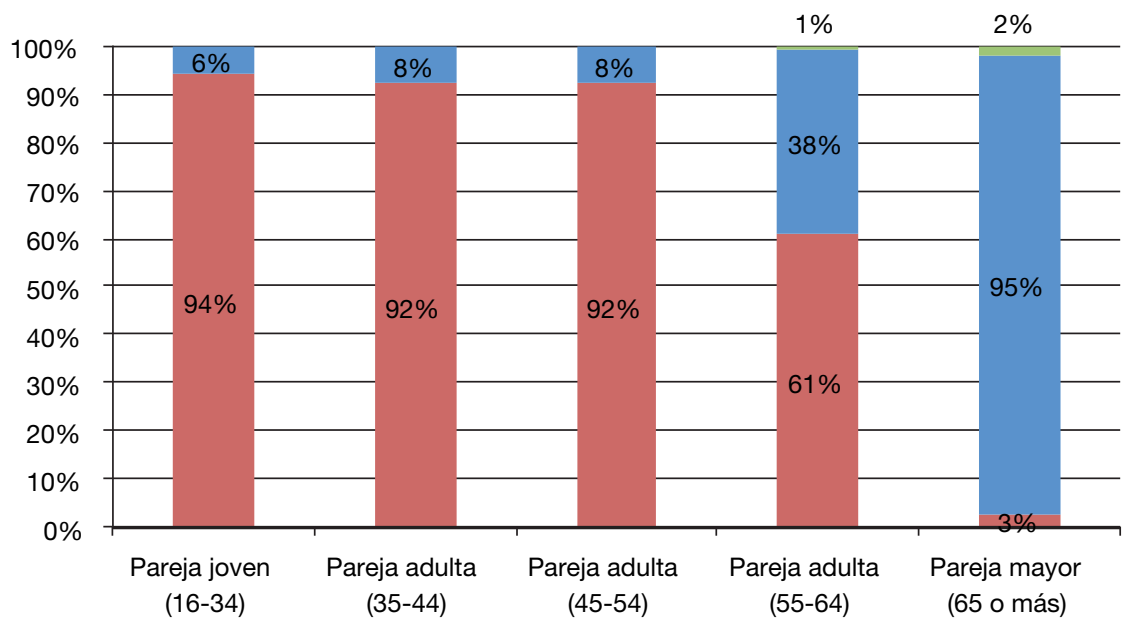

Hombres que nunca han estado empleados
Hombres actualmente sin empleo

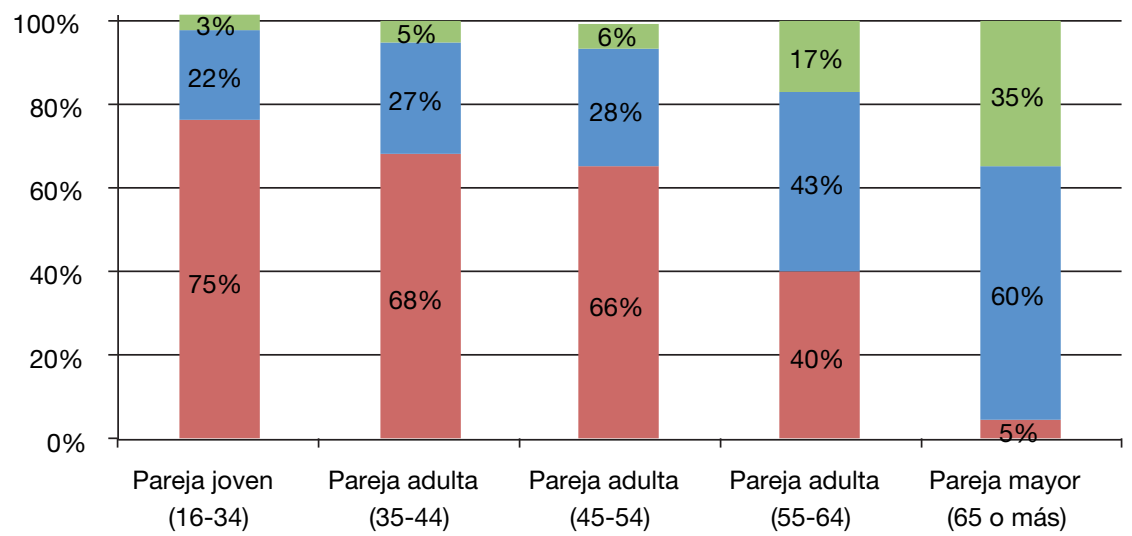

Mujeres que nunca han estado empleadas
Mujeres actualmente sin empleo

Hombres empleados

Fuente: elaboración propia a partir de los microdatos de la ECV 2008. 
social ${ }^{12}$ ) todavía prevalentes en nuestra sociedad. Esta diferencia en la participación de cada uno de los miembros de la pareja en el empleo es un factor muy importante para entender las relaciones de género y la persistencia de la división de roles en el seno de la pareja. Los hombres tienen una tasa de actividad laboral mayor que las mujeres, sobre todo hasta los 54 años de edad, como se puede ver en la figura 5, mientras que las mujeres de menos de 54 años tienen una participación en el mercado laboral superior al 65\% y, en el grupo de edad de 16 a 34, el $76 \%$ de las mujeres estaban empleadas en el momento de la realización de la encuesta, frente al 94\% de los hombres de la misma edad.

Esta diferencia en la actividad laboral se corresponde con una mayor implicación de las mujeres en las actividades domésticas y de cuidados. Como señala la última Encuesta de empleo del tiempo 2009-2010 (INE, 2011), en su conjunto, las mujeres dedican cada día dos horas y cuarto más que los hombres a las tareas del hogar. No obstante, en siete años, los varones han recortado esta diferencia en 41 minutos.

\section{Desigualdades económicas en las parejas de doble ingreso. La dependencia intrapareja}

Como corresponde a las diferencias señaladas respecto a la actividad laboral de mujeres y hombres, en los hogares analizados, hay menos mujeres que perciben ingresos y, si comparamos los ingresos de unas y otros, ellas ganan menos que ellos. Para medir esta desigualdad, hemos utilizado el planteamiento de Sorensen y McLanahan (1987), que proponen un indicador de dependencia económica intrapareja, entendiendo que una persona es dependiente económicamente cuando sus ingresos son inferiores, en un $15 \%$ o más, a los de su pareja. Nosotras hemos preferido evitar, en lo posible, el término dependiente, por sus varias connotaciones y porque debería ir acompañado de otro indicador de dependencia vital que todavía no está elaborado. Sin embargo, nos parece que una diferencia de ingresos de más del $15 \%$ es una buena medida de desigualdad.

La figura 6 muestra que las mayores desigualdades económicas se dan en las parejas de mayor edad ${ }^{13}$. Se observa claramente que, a medida que aumenta la edad, aumenta también el porcentaje de varones que aportan más al hogar, al tiempo que disminuye el porcentaje de mujeres que aportan más o el de quienes aportan por igual. Así, en el 80\% de las parejas mayores de 65 años, la

12. En física, se entiende por histéresis la cualidad de un material de mantener las alteraciones que le ha producido una fuerza externa, aún cuando dicha fuerza haya desaparecido. Hoy en día, las mujeres se han capacitado y están en condiciones de disponibilidad para cumplir cualquier función social, laboral o política en los mismos términos que los varones, pero nuestra sociedad, afectada por una especie de histéresis patriarcal, sigue pensando que no están suficientemente capacitadas o disponibles.

13. Para simplificar el análisis, y puesto que presentan datos prácticamente iguales, se han combinado las categorías de edad de 35 a 44 y de 45 a 54. 
Figura 6. Diferencias de ingresos superiores al 15\%, según la edad de la pareja

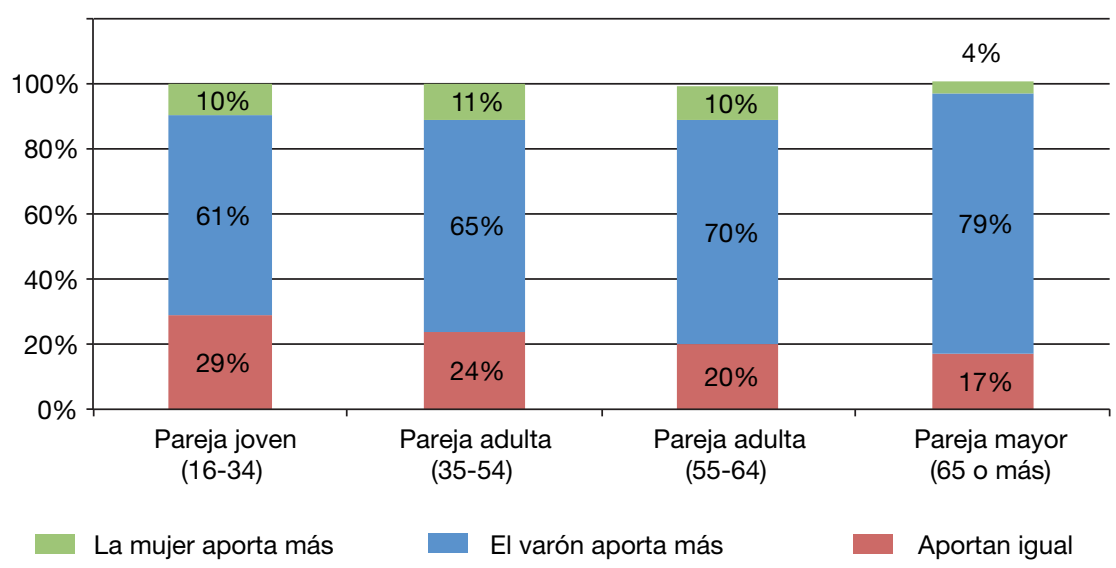

Fuente: elaboración propia a partir de los microdatos de la ECV 2008.

diferencia de ingresos a favor del varón es superior al $15 \%$. Este porcentaje va disminuyendo con la edad, pero no de forma muy notable, ya que, en el $61 \%$ de las parejas jóvenes, la diferencia de ingresos (de más del 15\%) también es a favor del varón. Así pues, la constante es que ellos ganan más en todas las generaciones.

Las parejas con ingresos similares, entendiendo como tales aquellas en las que la diferencia de ingresos entre el varón y la mujer no alcanza el 15\%, oscilan entre el 17\% en las parejas mayores de 65 años y el 29\% en las parejas jóvenes. A pesar de que, como se ha dicho, la desigualdad de ingresos entre hombres y mujeres se reduce ligeramente en la generación más joven, la diferencia de ingresos a favor del varón sigue siendo la norma.

Por el contrario, el porcentaje de parejas en las que las mujeres ganan (por encima del 15\%) más que los varones es muy reducido. En las parejas mayores de 65 años, este porcentaje no llega al 4\%; en las tres categorías adultas, oscila entre un $10 \%$ y un $12 \%$, y en las parejas jóvenes, está en un $9 \%$. Estos datos indican que, todavía hoy, pocas mujeres jóvenes consiguen empleos mejor pagados que los de sus parejas y que esta situación apenas mejora a largo de la edad activa.

Un análisis más pormenorizado de las diferencias de aportación económica entre hombres y mujeres ha puesto de manifiesto que, en la mitad de las parejas mayores, la diferencia entre la contribución del hombre y la de la mujer es superior al 90\%, lo que coloca a esta última en una situación de extrema dependencia económica respecto a su compañero. En las parejas más jóvenes, aunque las que presentan diferencias superiores al $90 \%$ se reducen, todavía constituyen un $13 \%$. Por el contrario, apenas encontramos hogares en cual- 
Tabla 2. Porcentaje de parejas con diferencias de ingresos superiores al $15 \%$ a favor del hombre, en función del nivel educativo y la situación laboral de las mujeres

\begin{tabular}{lc}
\hline \multicolumn{2}{c}{ Nivel educativo de las mujeres } \\
\hline Educación primaria o sin estudios & $61 \%$ \\
Educación secundaria obligatoria & $53 \%$ \\
Educación secundaria superior y FP & $38 \%$ \\
Educación universitaria & $20 \%$ \\
\hline \multicolumn{2}{c}{ Situación laboral de las mujeres } \\
\hline Empleada & $16 \%$ \\
Desempleada & $48 \%$ \\
Jubilada & $20 \%$ \\
\hline
\end{tabular}

Fuente: elaboración propia a partir de los microdatos de la ECV 2008.

quier tramo de edad en los que la contribución femenina sea superior al 16\% de los ingresos masculinos.

La desigualdad económica en el seno de las parejas está claramente ligada al nivel de estudios y a la situación laboral de las mujeres. A mayor nivel académico, mayor igualdad económica en el interior del hogar. Como se pone de manifiesto en la tabla 2, el $61 \%$ de las mujeres con estudios primarios o sin estudios tienen unos ingresos inferiores (en un 15\% o más) a los de sus parejas, frente al $20 \%$ de las que poseen estudios superiores, una cifra que, no obstante, continúa siendo muy elevada.

Y, como cabía esperar, si las mujeres tienen un empleo, la desigualdad económica entre los integrantes de la pareja es menor: el 16\% de las empleadas y el $20 \%$ de las mujeres jubiladas ganan menos que sus parejas, un porcentaje que alcanza el $48 \%$ en el caso de las desempleadas.

Volviendo a las diferencias de ingresos a favor de las mujeres, el hecho de que el fenómeno sea minoritario no implica que no merezca la pena estudiarlo con más detalle, porque un incremento del número de mujeres que ganen más que sus parejas resultaría, sin duda, un indicador de mayor igualdad social. ¿Qué factores pueden explicar mejor la probabilidad de que, en una pareja de doble ingreso, las mujeres ganen más que los hombres? Para poder contestar a esta pregunta, se ha llevado a cabo un análisis de regresión logística binaria ${ }^{14}$ entre las parejas de doble ingreso, en las que se han eliminado aquellas en las que ambos miembros ganan lo mismo, para poder comparar directamente los casos en las que unas ganan más que otros. Se han considerado variables que pudieran ser determinantes en la explicación de la probabilidad de que las mujeres aporten más ingresos que los hombres, como el tipo y tenencia de la

14. Análisis de regresión logística binaria sobre 2.613 hogares para predecir que las mujeres ganen más que los hombres. En los dos modelos calculados, el test del modelo completo contra el de la constante (chi cuadrado) es estadísticamente significativo, lo cual indica que las variables independientes predicen adecuadamente la probabilidad de la variable dependiente. 
Tabla 3. Análisis de regresión logística binaria para predecir la probabilidad de que la mujer gane más que el hombre

\begin{tabular}{|c|c|c|c|c|}
\hline & \multicolumn{2}{|c|}{ Modelo reducido } & \multicolumn{2}{|c|}{ Modelo completo } \\
\hline & Coef. B & $\operatorname{Exp}(B)$ & Coef. B & $\operatorname{Exp}(\mathrm{B})$ \\
\hline \multicolumn{5}{|l|}{ Edad del hombre (ref.: 65 años o más) } \\
\hline Menos de 44 años & $-0,312^{\star \star}$ & 0,732 & $-0,012$ & 0,988 \\
\hline De 45 a 65 años & $0,152^{\star \star}$ & 1,164 & $0,617^{\star \star}$ & 1,853 \\
\hline \multicolumn{5}{|l|}{ Edad de la mujer (ref.: 65 años o más) } \\
\hline Menos de 44 años & $1,385^{\star \star}$ & 3,996 & $1,445^{\star \star}$ & 4,242 \\
\hline De 45 a 65 años & $1,476^{\star \star}$ & 4,377 & $1,418^{\star \star}$ & 4,130 \\
\hline \multicolumn{5}{|l|}{ Situación laboral del hombre (ref.: otra inactividad) } \\
\hline Ocupado & $-1,730^{\star \star}$ & 0,177 & $0,331^{\star \star}$ & 1,393 \\
\hline En paro & $-0,005$ & 0,995 & $0,720^{\star \star}$ & 2,054 \\
\hline Estudiante & 20,201 & 1897,327 & 19,90 & 1897,327 \\
\hline Jubilado & $-0,596^{\star \star}$ & 0,551 & $-0,462^{\star \star}$ & 0,630 \\
\hline \multicolumn{5}{|l|}{ Situación laboral de la mujer (ref.: otra inactividad) } \\
\hline Ocupada & $3,349^{\star \star}$ & 28,484 & $2,153^{\star \star}$ & 8,610 \\
\hline En paro & $1,946^{\star \star}$ & 6,999 & $1,468^{\star \star}$ & 4,340 \\
\hline Estudiante & $-16,122$ & 278,749 & $-16,331$ & 268,368 \\
\hline Jubilada & $2,720^{\star \star}$ & 15,184 & $2,977^{\star \star}$ & 19,620 \\
\hline \multicolumn{5}{|l|}{ Nivel educativo del hombre (ref.: educación superior) } \\
\hline No ha recibido nunca educación & $1,812^{\star \star}$ & 6,121 & $1,287^{\star \star}$ & 3,622 \\
\hline Educación primaria & $0,730^{\star \star}$ & 2,075 & $0,596^{\star \star}$ & 1,816 \\
\hline Educación secundaria 1. a etapa & $0,714^{\star \star}$ & 2,043 & $0,523^{\star \star}$ & 1,687 \\
\hline Educación secundaria 2 . $^{\mathrm{a}}$ etapa & $0,569^{\star \star}$ & 1,767 & $0,596^{\star \star}$ & 1,814 \\
\hline FP o inserción con título de ESO & $-0,283^{\star \star}$ & 0,767 & $-0,220^{\star \star}$ & 0,802 \\
\hline \multicolumn{5}{|l|}{ Nivel educativo de la mujer (ref.: educación superior) } \\
\hline No ha recibido nunca educación & $-2,291^{\star *}$ & 0,101 & $-2,455^{\star \star}$ & 0,086 \\
\hline Educación primaria & $-1,768^{\star *}$ & 0,171 & $-2,192^{\star *}$ & 0,112 \\
\hline Educación secundaria 1. a etapa & $-1,591^{\star \star}$ & 0,204 & $-1,704^{\star \star}$ & 0,182 \\
\hline Educación secundaria 2 . $^{\text {e }}$ etapa & $-1,128^{\star \star}$ & 0,324 & $-1,280^{\star \star}$ & 0,278 \\
\hline FP o inserción con título de ESO & $-1,031^{\text {** }}$ & 0,357 & $-1,534^{\star \star}$ & 0,216 \\
\hline Riesgo de pobreza & & & $-0,991^{\star \star}$ & 0,371 \\
\hline \multicolumn{5}{|c|}{ Tipo de vivienda (ref.: piso o apto. en edif. de diez o más viviendas) } \\
\hline Vivienda unifamiliar independiente & & & $0,341^{\star \star}$ & 1,407 \\
\hline Vivienda unifamiliar adosada o pareada & & & $0,284^{\star \star}$ & 1,329 \\
\hline Piso o apto. en edif. de menos de diez viviendas & & & $0,470^{\star \star}$ & 1,600 \\
\hline \multicolumn{5}{|l|}{ Tenencia de la vivienda (ref.: cesión gratuita) } \\
\hline En propiedad & & & $-0,112^{\star \star}$ & 0,894 \\
\hline En alquiler pagando un precio de mercado & & & $-0,097^{\star \star}$ & 0,907 \\
\hline En alquiler pagando un precio de mercado reducido & & & $0,100^{\star \star}$ & 1,105 \\
\hline \multicolumn{5}{|l|}{ Pareja según ocupación (ref.: ninguno ocupado) } \\
\hline Él ocupado y ella no & & & $-1,970^{\star \star}$ & 0,139 \\
\hline Ambos ocupados & & & $0,043^{\star \star}$ & 0,958 \\
\hline Ella ocupada y él no & & & $2,472^{\star \star}$ & 11,841 \\
\hline \multicolumn{5}{|l|}{ Edad de la pareja (ref: pareja mayor (65 años o más) } \\
\hline Pareja joven (16-34) & & & $-0,671^{\star \star}$ & 0,511 \\
\hline Pareja adulta (35-44) & & & $-0,553^{\star \star}$ & 0,575 \\
\hline Pareja adulta (45-54) & & & $-0,657^{\star \star}$ & 0,519 \\
\hline Pareja adulta (55-64) & & & $-0,550^{\star \star}$ & 0,577 \\
\hline Constante & $-3,976^{\star \star}$ & 0,027 & $-3,364^{\star \star}$ & 0,035 \\
\hline-2 log de la verosimilitud & 3.342 .535 & & 2.904 .122 & \\
\hline$R$ cuadrado de Nagelkerke & 0,323 & & 0,409 & \\
\hline$\%$ global de casos correctamente pronosticados & $88,2 \%$ & & $89,6 \%$ & \\
\hline
\end{tabular}

${ }^{\star *} p<0,00$ 
vivienda, el nivel educativo, la renta neta del hogar, así como la actividad, la ocupación y la nacionalidad de los miembros de la pareja.

Se ha trabajado con dos modelos de regresión logística, cuyos resultados se incluyen en la tabla 3: uno completo, que incluye variables relativas a las parejas, el hogar y sus miembros, y otro más reducido, que incluye, fundamentalmente, variables individuales. Los datos ponen de manifiesto que la mayor parte de las categorías de las variables consideradas son significativas ${ }^{15}$ y que es preferible trabajar con el modelo más completo, ya que arroja mejores resultados $^{16}$. Con la excepción de los hombres menores de 44 años, y las mujeres y los hombres que son estudiantes, el resto de variables presentan coeficientes de regresión significativos.

Considerando los $\exp (\mathrm{B})$ de signo positivo y fijándonos en el modelo completo, podemos afirmar que las categorías consideradas que tienden a aumentar la probabilidad de que las mujeres ganen más que los hombres son, fundamentalmente, las formadas por aquellas parejas que muestran alguna de las tipologías siguientes: hombres mayores de 45 años, mujeres menores de 65, hombres en paro y mujeres ocupadas o jubiladas, que, dentro de la pareja, ella esté ocupada y él no, que el hombre tenga un nivel educativo bajo y que vivan en un piso o en un apartamento de un edificio de menos de diez viviendas y paguen por la misma un precio reducido de mercado. Parece que las mujeres tienden a igualarse e incluso a gozar de una posición económica mejor que sus compañeros varones en hogares que viven situaciones de mayor precariedad, dado que las características mencionadas, como el hecho de que los varones tengan un nivel educativo bajo, o que estén en el paro, e incluso que vivan en pisos o apartamentos frente a viviendas unifamiliares, se dan en mayor medida en parejas con bajos salarios.

\section{Desigualdades económicas entre hombres y mujeres en otros tipos de hogares}

Una vez analizadas las diferencias económicas que se producen en las parejas de doble ingreso, tiene interés comparar su situación con la realidad de hombres y mujeres en el resto de hogares. Los datos de la ECV que se presentan en la tabla 4 muestran que, en todos los modelos de familia que hemos analizado, hay menos mujeres perceptoras de ingresos que varones, y que aquellas que

15. Las categorías de referencia que no están presentes en la ecuación, dado que son las categorías de contraste, se resaltan en negrita. El estadístico de Wald mide la significatividad de cada coeficiente $\mathrm{B}\left({ }^{* *}: p<0,000\right)$. Los $\exp (\mathrm{B})$ indican que, cuando se incrementa o se disminuye cada variable independiente en una unidad, la probabilidad de las mujeres de ganar más que los hombres (variable dependiente) tiene una probabilidad $x$ veces mayor o menor. Un valor superior a 1 indica que la probabilidad aumenta; un valor inferior a 1 , que disminuye. Si al exponente se le resta 1 y se multiplica por 100, se obtiene el porcentaje en que aumenta o disminuye la variable dependiente.

16. La R cuadrado de Nagelkerke, que equivale al porcentaje de varianza explicada por el modelo, es de un $41 \%$ frente a un $32 \%$. Asimismo, el porcentaje global de casos correctamente explicados es de casi un 90\%, y el modelo completo reduce mejor el -2log de la verosimilitud. 
Tabla 4. Ingresos brutos totales medios por sexo, edad y tipo de hogar*

\begin{tabular}{lcr} 
& \multicolumn{2}{c}{ Ingresos brutos totales de la persona } \\
\cline { 2 - 3 } & Hombre & Mujer \\
\hline Hogares con parejas: & $22.900 €$ & $11.181 €$ \\
En edad laboral & $13.803 €$ & $3.352 €$ \\
Mayor de 65 años & & $17.441 €$ \\
\hline Hogares unipersonales: & $19.096 €$ & $9.260 €$ \\
En edad laboral & $13.055 €$ & \\
Mayor de 65 años & & $12.650 €$ \\
En edad laboral & &
\end{tabular}

* Diferencias de medias significativas para $p<0,01$ en todos los casos (test Anova).

1. Dado que apenas existen hogares monoparentales encabezados por varones, no se les ha podido incluir en la comparación.

Fuente: elaboración propia a partir de los microdatos de la ECV 2008.

perciben ingresos, vivan solas o en pareja, lo hacen en una cuantía inferior a la de los hombres en situación comparable.

Las mujeres que viven en pareja en edad laboral tienen unos ingresos sustancialmente menores $(11.181 €)$ que aquellas que no viven en pareja $(17.441 €)$, aunque siempre inferiores a los de los varones en su misma situación. De la misma forma, las que encabezan hogares monoparentales perciben menos que los hombres en cualquiera de los modelos analizados e incluso menos que las mujeres que viven en hogares unipersonales.

Por su parte, los varones que tienen mayores ingresos son aquellos en edad laboral que viven en pareja $(22.900 €)$, seguidos de los varones que viven solos (19.096€ anuales).

Las personas mayores de 65 años, por su parte, y como cabe esperar, perciben, en los tipos de hogares estudiados, menos ingresos que las personas en edad laboral y se mantienen las diferencias a favor de los hombres en mayor medida que en las edades laborales.

Los datos de situación económica unidos a los de desigualdad de género en el interior de los hogares ponen de manifiesto, por tanto, que hay que prestar una atención especial a dos colectivos especialmente vulnerables: las mujeres mayores que viven solas y aquellas que tienen a su cargo menores y son cabezas de hogar.

Aunque es previsible que, en estos años de profunda crisis, sus perspectivas no hayan hecho más que empeorar, no debe olvidarse, sin embargo, que la situación tanto de los hogares con parejas como de los monoparentales en edad laboral tampoco está exenta de bordear situaciones de riesgo de pobreza, dados sus bajos ingresos y las condiciones de creciente precariedad del mercado de trabajo. Resultaría de gran interés, sin duda, analizar, en el futuro, las desigualdades de género que se producen en las parejas que pudieran constituir este nuevo colectivo de «trabajadores pobres» (Gutiérrez Palacios y García Espejo, 2010). 


\section{Conclusiones}

El análisis realizado sobre la propiedad del dinero y la distribución de los ingresos en los hogares españoles nos ha revelado una tendencia hacia una mayor igualdad entre hombres y mujeres, sobre todo en las generaciones más jóvenes. Sin embargo, también hemos podido observar cómo persisten ciertos elementos vinculados a las desigualdades de género.

Por un lado, la mayoría de las parejas jóvenes tiene doble ingreso, frente al modelo tradicional de varón proveedor y mujer ama de casa, que sigue siendo el dominante en los hogares de las generaciones mayores, pero que pierde presencia en nuestros días. Este fenómeno está relacionado con la mejora del nivel educativo de las mujeres en las últimas décadas y con su acceso al mercado laboral. $\mathrm{Si}$ bien el conjunto de la población española ha mejorado sustancialmente su nivel educativo en las últimas décadas, en el caso femenino, la mejora ha sido mayor, de manera que, por primera vez en la historia, las mujeres más jóvenes (menores de 35 años) tienen niveles educativos más elevados que los de sus parejas. Otro de los factores que, en principio, favorece la igualdad en las relaciones de género es la incorporación de las mujeres al trabajo remunerado. Las jóvenes presentan unas altas tasas de actividad y sólo un porcentaje muy residual no ha trabajado nunca de forma remunerada, lo que supone un cambio espectacular con la situación laboral de las mujeres de las generaciones mayores (más de 55 años).

Por otro lado, hemos podido observar que, a pesar del aumento de las parejas de doble ingreso, ellas ganan menos que sus compañeros varones en todas las generaciones. El balance económico es favorable a los hombres, esto es, el predominio económico masculino en los hogares es la norma, por su cuantía y generalización. Un fenómeno que apunta a que las mujeres apenas consiguen empleos mejor pagados que los de sus parejas, ni siquiera las más jóvenes, que, como decíamos, están mejor formadas que los varones. De hecho, el análisis de los modelos de regresión logística realizados muestra que ellas tienden a ganar más que sus parejas cuando los varones tienen un nivel educativo bajo, o cuando ellas están ocupadas y ellos no, o cuando se dan otras circunstancias que nos llevan a pensar en parejas de salarios bajos. Estas diferencias en los ingresos sitúan a las mujeres en una posición de partida peor a la hora de utilizar el dinero, tanto para sus gastos personales, como para negociar con sus parejas gastos del hogar, como hemos puesto de manifiesto en otras investigaciones previas (Díaz et al., 2004, 2007; Dema, 2006, 2009; Dema y Díaz, 2010).

Finalmente, si comparamos la situación de mujeres y varones que viven en pareja con otros tipos de modelos familiares, resulta que, en todos ellos, hay menos mujeres que varones perceptoras de ingresos, y aquellas que sí los perciben reciben una cuantía inferior a la de los varones en situación comparable. No obstante, las mujeres que viven en pareja y que están en edad laboral reciben unos ingresos un 36\% inferiores a las que viven solas y un $12 \%$ inferiores a las que encabezan hogares monoparentales, lo contrario de lo que les sucede a los varones que viven emparejados y en edad laboral, que son quienes más ingresos perciben, un $17 \%$ por encima de los que viven solos. 


\section{Referencias bibliográficas}

Alberdi, Inés (1999). La nueva familia española. Madrid: Taurus.

- (2006). «La transformación de las familias en España». Ábaco, 49-50, 29-65.

Blinder, Alan S. (1973). «Wage Discrimination: Reduced Form and Structural Estimates». The Journal of Human Resources [en línea], 8 (4), 436-455. $<$ http://dx.doi.org/10.2307/144855>.

Blood, Robert y Wolfe, Donald (1960). Husbands and Wives. Nueva York: Free Press.

Cabo, Gema de y Garzón, M. a José (2007). Diferencia y discriminación salarial por razón de sexo. Madrid: Instituto de la Mujer. Serie Estudios, 100.

Campo, Salustiano del (1991). La «nueva» familia española. Madrid: Eudema.

Carrasco, Cristina (2003). "La sostenibilidad de la vida humana: ¿un asunto de mujeres?». Utopias, 195, 151-173.

Carrasco, Cristina y Rodríguez, Arantxa (2000). "Women, Families, and Work in Spain: Structural Changes and New Demands». Feminist Economics [en línea], 6 (1), 45-57. <http://dx.doi.org/10.1080/135457000337660>.

Clarke, Victoria; Burgoyne, Carole y Burns, Maree (2005). «For love or money?: Comparing lesbian and gay, and heterosexual relationships». The Psychologist, 18 (6), 356-358.

DALY, Mary y LewIS, Jean (2000). «The concept of social care and the analysis of contemporary welfare states». British Journal of Sociology [en línea], 51 (2), 281-298. <http://dx.doi.org/10.1111/j.1468-4446.2000.00281.x>.

Dema Moreno, Sandra (2006). Una pareja, dos salarios: El dinero y las relaciones de poder en las parejas de doble ingreso. Madrid: CIS.

- (2009). «Behind the Negotiations: Financial Decision-Making Processes in Spanish Dual-Income Couples». Feminist Economics [en línea], 15 (1), 27-56. <http://dx.doi.org/10.1080/13545700802620575>.

- (2011). «Tensions Between Work, Family and Personal Life in Spanish Dual-Income Couples». En: Drobnic, Sonja y Guillén, Ana (eds.). Work-Life Balance in Europe: The Role of Job Quality. Houndmills, Basingstoke, Hampshire: Palgrave Macmillan, 231-251.

Dema Moreno, Sandra y Díaz Martínez, Capitolina (2010). "Gender inequalities and the role of money in Spanish dual-income couples». European Societies [en línea], 12 (1), 65-84. <http://dx.doi.org/10.1080/14616690903219181>.

Díaz Martínez, Capitolina; Dema Moreno, Sandra e Ibáñez Pascual, Marta (2004). «El papel del dinero en las relaciones de pareja y familia». En: Monreal, Juan; Díaz Martínez, Capitolina y García Escribano, Juan José (eds.). Viejas sociedades, nueva sociología. Madrid: CIS, 233-254.

- (2007). "The Intertwining of Money and Love in Couple Relationship». En: Stocks, Janet; Díaz Martínez, Capitolina y Hallerod, Björn (eds.). Modern couples, sharing money, sharing life. Nueva York: Palgrave McMillan, 100-142.

Franco, Ana y WinQvist, Karin (2002). "Women and men reconciling work and family life». Statistics in focus: Population and Social Conditions. European Communities: Eurostat.

González, Juan Jesús y Requena, Miguel (2005). Tres décadas de cambio social en España. Madrid: Alianza Editorial.

Gutiérrez Palacios, Rodolfo y García Espejo, Isabel (2010). «Empleo y pobreza en España». Panorama Social, 12, 29-40. 
INE (2011). Encuesta de empleo del tiempo 2008-2010 [en línea]. Madrid. INE. $<$ http://www.ine.es/jaxi/menu.do?L=0\&type=pcaxis\&path=\%2Ft25\%2Fe447\&file $=$ inebase $>$.

INJUVE (2008). «Jóvenes e igualdad de género». Estudio INJUVE 135 [en línea]. Madrid. Ministerio de Sanidad, Servicios Sociales e Igualdad. <http://www.injuve.es/ca/observatorio/familia-pareja-e-igualdad-de-genero/ jovenes-e-igualdad-de-genero>.

Jurado Guerrero, Teresa y Naldini, Manuela (1996). «Is the South so Different?: Italian and Spanish Families in Comparative Perspective». South European Society and Politics [en línea], 1, 42-66.

<http://dx.doi.org/10.1080/13608749608539482>.

LARrAÑAGA, Isabel et al. (2008). "Impacto del cuidado informal en la salud y la calidad de vida de las personas cuidadoras: Análisis de las desigualdades de género». Gaceta Sanitaria [en línea], 22 (5), 443-450. <http://dx.doi.org/10.1157/13126925>.

Meil Landwerlin, Gerardo (1999). La posmodernización de la familia española. Madrid: Acento Editorial.

Neumark, David (1988). "Employers' Discriminatory Behavior and the Estimation of Wage Discrimination». The Journal of Human Resources [en línea], 23 (3), 279-295. <http://dx.doi.org/10.2307/145830>.

OAxacA, Ronald (1973). "Male-female wage differentials in urban labor markets». International Economic Review [en línea], 14 (3), 693-709. $<$ http://dx.doi.org/10.2307/2525981>.

PAHL, Jan (1989). Money and Marriage. Houndmills, Basingstoke, Hampshire y Londres: Macmillan.

Sallé Alonso, M. a Ángeles y Molpeceres Álvarez, Laura (2010) (coord.). La brecha salarial: realidades y desafíos: Las desigualdades salariales entre mujeres y hombres España 2009. Madrid: Ministerio de Igualdad y Ministerio de Trabajo e Inmigración. Colección Economía Mujer Empresa.

Sorensen, Annemette y Mclanahan, Sara (1987). «Married Women’s Economic Dependency: 1950-1980». American Journal of Sociology [en línea], 92, 659-687. <http://dx.doi.org/10.1086/228792>.

Stocks, Janet; Díaz Martínez, Capitolina y Hallerod, Björn (2007) (eds.). Modern couples, sharing money, sharing life. Nueva York: Palgrave Macmillan.

Tichenor, Veronica (1999). "Status and income as gendered resources: The case of marital power». Journal of Marriage and the Family [en línea], 61, 638-650. <http://dx.doi.org/10.2307/353566>.

Toвío, Constanza (2005). Madres que trabajan. Madrid: Cátedra.

Torns, Teresa (2005). «De la imposible conciliación a los permanentes malos arreglos». Cuadernos de Relaciones Laborales, 23, 15-33.

Torns, Teresa y Recio, Carolina (2013). "Género, trabajo y vida económica». En: Díaz Martínez, Capitolina; Dema Moreno, Sandra. Sociología y género. Madrid: Tecnos, 153-200.

Villar, Antonio (2010) (dir.). Mujeres y mercado laboral en España: Cuatro estudios sobre la discriminación salarial y la segregación laboral. Bilbao: Fundación BBVA.

Vogler, Carolyn y PAHL, Jan (1994). «Money, Power, Inequality within Marriage». The Sociological Review [en línea], 42 (2), 263-288. <http://dx.doi.org/10.1111/j.1467-954X.1994.tb00090.x>.

Zelizer, Viviana (1997). The Social Meaning of Money. Princeton: Princeton University Press. 